\title{
Сложные задачи поверхностного монтажа и гибкие решения Europlacer
}

\author{
А. Гаранин ${ }^{1}$
}

Современная экономическая ситуация устанавливает всё более жесткие требования по отношению к техническим возможностям и эффективности работы сборочно-монтажного оборудования отечественных предприятий. В статье проанализированы условия, которым должно соответствовать конкурентоспособное сборочно-монтажное производство, и приведены примеры эффективных технических решений для различных аспектов сборки электронных узлов по технологии поверхностного монтажа.

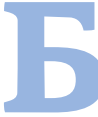

лагодаря государственной поддержке сборочно-монтажные линии поверхностного монтажа на большом числе отечественных предприятий были оснащены высокопроизводительным современным оборудованием, и сегодня многие российские компании обладают возможностью производить крупные серии изделий электронной техники. Чаще всего на отечественных производствах можно встретить высокопроизводительные автоматические установщики компонентов, изготовленные в Японии или Южной Корее. Такие станки, изначально сконструированные для применения в условиях массового производства Китая и стран Юго-Восточной Азии, обладают паспортной скоростью сборки до 80 тыс. комп. /4, а линии на их основе рассчитаны на сборку десятков тысяч изделий в месяц (рис. 1) Они предназначены для работы с однородной компонентной базой, качественной стандартизированной упаковкой и печатными платами определенной толщины, формы и габаритов.

Однако, по данным исследований, в промышленно развитых странах крупносерийное и массовое производства составляют лишь 20\%, а единичное, мелкосерийное и серийное - 80\%. [1]. Такая же ситуация складывается и в России. Отличительной стороной отечественной электронной отрасли является преобладание мелкосерийного и среднесерийного многономенклатурного типа производства с большей долей ручного труда. На многих предприятиях машиностроения механизировано не более 20-25\% сборочных операций и только около 5\% - автоматизировано. В приборостроении и в электронной промышленности эти цифры несколько выше, но и они неутешительны: уровень механизации не превышает 3035\%, а автоматизации - 10-15\% [1].

Разумеется, уровень автоматизации при сборке электронных узлов должен соответствовать современным

ООО «новые Технологии». требованиям. Продолжающаяся миниатюризация, применение современных электронных компонентов и увеличение сложности продукции задает критерии для автоматического оборудования. В любом случае, использование ручного труда при недостаточной автоматизации не позволяет изготавливать сложные изделия с требуемым качеством и производительностью.

Учитывая специфику российской электронной промышленности, можно определить пять основных задач, стоящих перед предприятием на пути кдостижению конкурентного преимущества на рынке:

1. Быстрая реакция. Необходимо обладать возможностью оперативно реагировать на изменение ситуации. Должна быть обеспечена возможность быстрого перехода с одного типа собираемого изделия на другой, подготовка сборочного участка должна занимать минимальное время.

2. Готовность к сборке любого вида изделий. Для обеспечения конкурентоспособности предприятия его производство должно быть готово к сборке печатных узлов любой толщины, размера, формы.

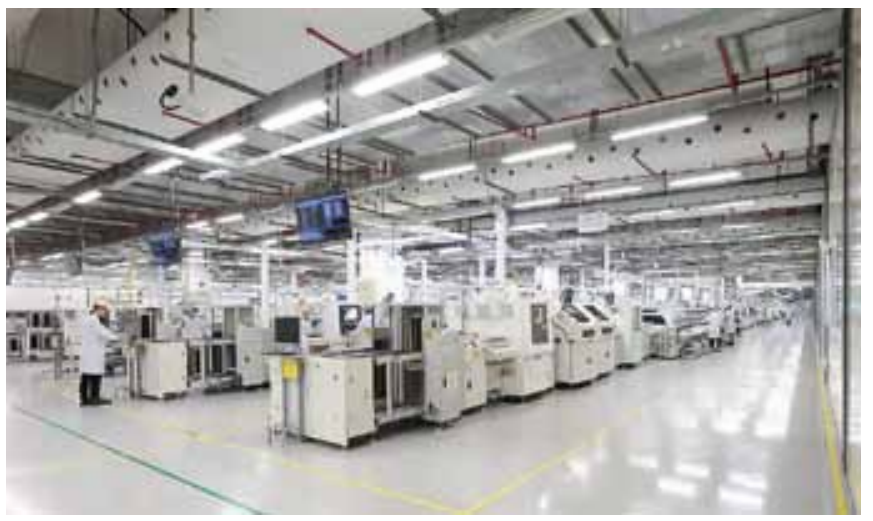

Рис. 1. Сборочная линия для массового производства электроники 
Оборудование должно работать с любыми видами электронных компонентов, включая отечественные.

3. Работа с неупакованными компонентами и обрезками ленты. Качество конечного изделия не должно зависеть от типа упаковки электронных компонентов. Автоматический установщик должен иметь возможность захватывать компоненты из любого типа носителей, работать с единичными компонентами и обрезками ленты.

4. Контроль качества установки компонентов. Помимо точности позиционирования в горизонтальной плоскости, для получения высокого качества монтажа важен автоматический контроль усилия при установке каждого отдельного компонента. Необходимо также обеспечить работу с печатными платами, подверженными изгибу и короблению.

5. Контроль компонентов: защита от брака, контрафакта и ошибок оператора. В оборудовании должна быть предусмотрена как защита от использования некачественных, контрафактных компонентов, так и устранение влияния человеческого фактора при подготовке производства.

Ниже рассмотрены возможные решения перечисленных задач на примере оборудования компании Europlacer.

\section{БОЛЬШЕ ВЫПОЛНЕННЫХ ЗАКАЗОВ ЗА ТО ЖЕ ВРЕМЯ}

При подготовке установщика к сборке нового изделия самое большое время затрачивается на зарядку компонентов в питатели, а также на обучение установщика высотам и точным координатам компонентов в питателях.

Основной особенностью автоматических установщиков компонентов, производимых французским предприятием Europlacer, является оригинальная конструкция установочной головы. Изначально разработанные для обеспечения максимальной гибкости в работе, все автоматы Europlacer оснащаются установочными головами, имеющими систему фиксации касания с обратной связью. Эта система непрерывно контролирует процесс монтажа компонента - от момента его захвата из питателя до установки с контролируемым усилием на запрограммированную позицию.

Благодаря автоматической системе контроля процесса захвата компонента время подготовки элементной базы для сборки нового изделия значительно сокращается. При зарядке компонента в питатель, при обучении установщика новому элементу нет необходимости измерять его высоту иии точно указывать уровень захвата из питателя. Система Europlacer делает это за оператора. При этом зарядка интелмектуального питателя muna Europlacer ii-Feed занимает менее $30 \mathrm{c}$.

\section{ГОТОВНОСТЬ К СБОРКЕ} ЛЮБОГО ВИДА ИЗДЕЛИЯ

На сегодня задача обеспечения качественного монтажа элементов на их посадочные места - одна из важнейших в технологии производства изделий радиоэлектронной аппаратуры и вычислительной техники

Проблему при монтаже электронных компонентов и интегральных микросхем представляют различные виды выводов компонентов, размеры выводов и шаг между ними. Ручной монтаж, в принципе позволяющий решить эту проблему, не обеспечивает должной производительности и качества, поскольку подвержен значительному влиянию субъективных факторов, провоцирующих появление брака; для компонентов же с шагом выводов 0,1 мм ручная пайка становится практически невозможной. Очевидно, что эти недостатки заставляют сокращать объемы ручного монтажа.

В современных сборочно-монтажных линиях элементы чаще всего устанавливаются двумя станками. Первыйвысокоскоростной автомат, предназначен для установки основной массы комплектующих: дискретных чип-компонентов и большинства микросхем. Важными его характеристиками является количество захватов для одновременной установки компонентов и наличие автоматизированного магазина с захватами под разные типы корпусов для их смены без остановки цикла монтажа изделия. Второй аппарат - высокоточный станок, предназначен для установки микросхем с малыми размерами выводов, компонентов в корпусах ВGА, монтажа по методу flip-chip, а также для установки нестандартных компонентов, например, разъемов [2].

По сравнению со стандартными решениями оборудование Europlacer изначально обладает возможностью работы со всеми видами электронных компонентов (рuс. 2, 3). Скорость работы, точность установки, а также большое количество питателей позволяют использовать один

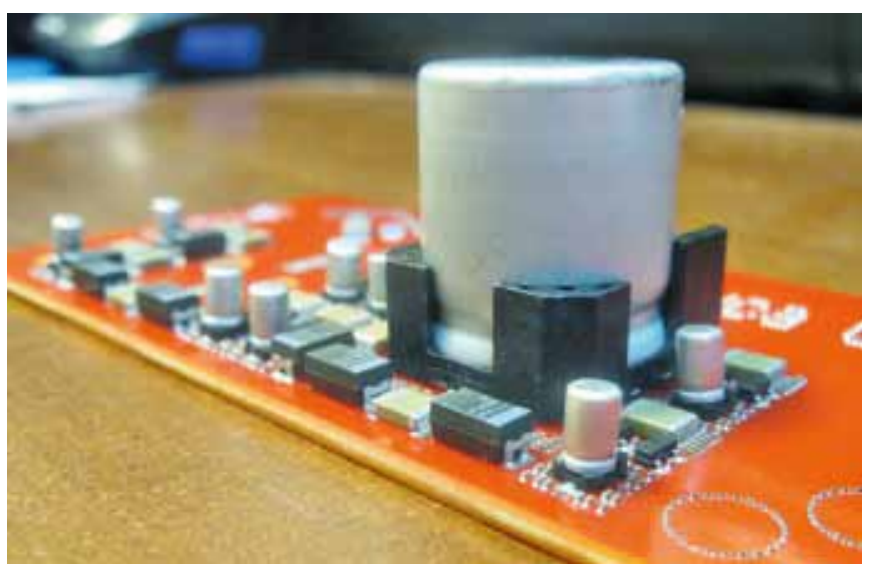

Рис. 2. Печатный узел с разнородной компонентной базой, собранный на установщике Europlacer iineo+ 


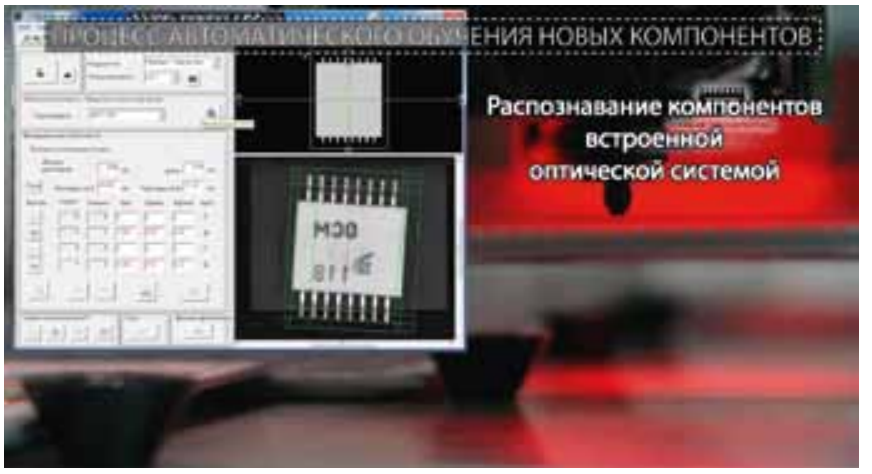

Рис. 3. Europlacer работает с отечественной ИМС

установщик Europlacer там, где другие производители предлагают два.

Размеры печатной платы и ее толщина для установщиков Europlacer фактически не ограничены. Cтандартная комплектация автомата позволяет работать с печатными платами дииной до 700 мм.

\section{НЕУПАКОВАННЫЕ КОМПОНЕНТЫ И ОБРЕЗКИ ЛЕНТЫ}

Часто производители электроники сталкиваются с ситуацией, когда необходимо изготовить несколько изделий в условиях ограниченного количества комплектующих (рис. 4). Изготовление единичных изделий, средних и малых серий не должно вызывать трудностей при подготовке производства. Однако при использовании высокоскоростных установщиков или установщиков, рассчитанных на работу со стандартизированной упаковкой компонентов, попытки работы с обрезками ленты

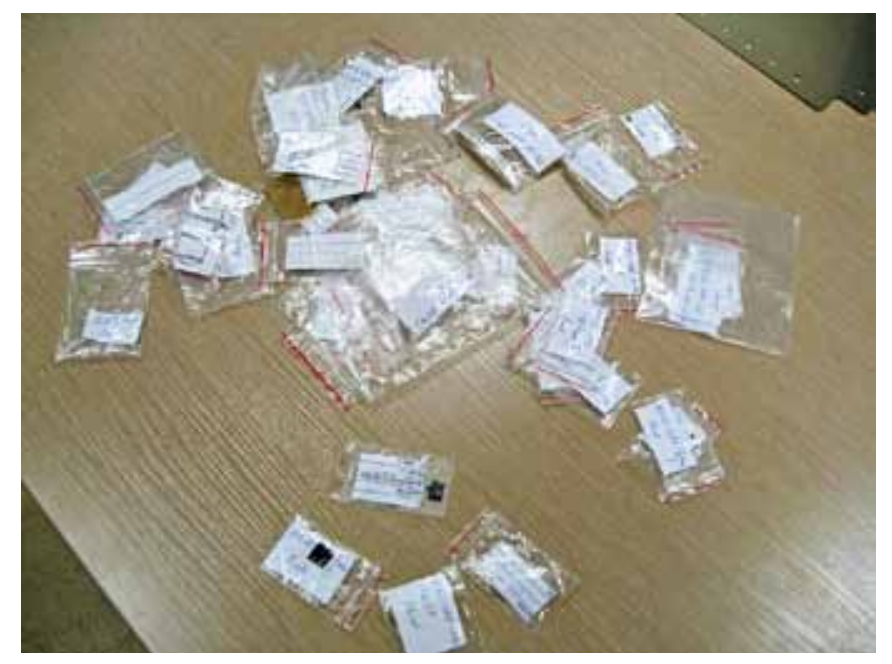

Рис. 4. Отечественным производителям электроники часто приходится получать комплектацию в таком виде - каждый компонент предоставляется точно в том количестве, которое нужно для выполнения заказа часто становятся непреодолимой проблемой: системы подачи чип-компонентов и интегральных микросхем не позволяют использовать нестандартную упаковку, а зарядка коротких обрезков ленты в питатель чаще всего невозможна.

Оборудование Europlacer не ставит ограничений при работе с разнородной упаковкой электронных компонентов. Интелпектуальные пuтатели Europlacer работают с обрезками иенты, причем их отпичительной особенностью является отсутствие необходимости оставиять технологический запас компонентов на зарядку.

\section{КОНТРОЛИРУЕМОЕ КАЧЕСТВО УСТАНОВКИ КОМПОНЕНТОВ}

Качественная установка компонентов на плату начинается с центрирования, для чего применяются лазерные системы позиционирования или системы технического зрения на основе камер высокого разрешения. Для многопортальных систем характерно использование видеокамер отдельно для каждой установочной головы. Типичные системы имеют станции центрирования с камерами нижнего обзора: установочная голова подводит компонент к видеосистеме, затем, после центрирования, перемещается в зону установки .

В роторных системах Europlacer осуществляется контроль и центрирование компонента при каждом повороте монтажной головы. Центрирующая видеосистема установлена непосредственно на установочной голове автомата. Такая компоновка позволяет устранить необходимость дополнительных затрат времени на перенос компонента в поле зрения видеосистемы.

особенно важно отметить, что конструкция системы видеоцентрирования Europlacer обеспечивает полный контроль выводов интегральных микросхем. Без дополнительных затрат любой установщик Europlacer обладает возможностью контролировать геометрические размеры выводов микросхем, проводить проверку на компланарность. Каждый вывод проверяется на дефекты в осях X, Y и Z

Проблема, с которой нередко приходится сталкиваться технологам сборочно-монтажного производства - деформация печатных плат после оплавления припоя во время сборки одной из сторон печатного узла. На практике в качестве предельно допустимого отклонения от плоскостности часто принимают величину, равную 0,5\% самого большого размера платы в горизонтальной плоскости. Автоматизированные методы производства накладывают более серьезные ограничения как на заготовки, находящиеся в процессе изготовления, так и на собранные печатные узлы. Например, загрузочные устройства автоматических линий не будут работать, если величина деформации заготовок превышает их номинальную толщину. Это означает, 
что для материала толщиной 1,5 мм независимо от размера заготовки допустимое отклонение от плоскостности не может быть более 1,5 мм. При длине заготовки 600 мм такое отклонение в относительных величинах составит 0,25\% [3], то есть ограничение получается вдвое более жестким, чем упомянутые 0,5\% максимального размера.

При ручной загрузке платы с ее одновременным закреплением требования по деформации, предъявляемые загрузочным устройством, снимаются. Однако в этом случае плата в процессе монтажа компонентов может находиться в напряженном состоянии, что впоследствии приведет к образованию механических напряжений в паяных соединениях

Для исправления деформации требуется нагрев материала печатной платы выше температуры стеклования, при этом фиксация платы должна осуществляться с учетом ее теплового расширения.

Деформации печатного узла чрезвычайно сложно устранить по следующим причинам:

- повышенные температуры могут негативно сказаться на компонентах;

- паяные соединения будут держать печатный узел в состоянии деформации, поэтому единственная эффективная мера - удаление компонентов до начала процесса исправления дефекта;

- может произойти растрескивание контактов, если перед попыткой распрямить жесткую плату паяные соединения не расплавить; в случае растрескивания плату, как правило, приходится утилизировать

Чаще всего производителю электроники по разным причинам приходится брать в работу искривленные печатные платы. Очень важно не упускать из виду, что деформации могут вызывать следующие отрицательные эффекты при автоматизированной установке компонентов:

- заклинивание печатных плат;

- потерю точности установки компонентов;

- возможность появления брака при установке компонентов;

- ухудшение способности оборудования распознавать реперные знаки;

- уменьшение срока службы захватов установщика;

- выход из строя точной механики и узлов перемещения установочных голов.

В оборудовании Europlacer реализована автоматическая система контроля за уровнем и усилием установки каждого компонента. Благодаря этой системе работа с искривленными печатными платами не является проблемой. Коррекция усипия при установке июбого компонента производится в автоматическом режиме без потери скорости с июбой возможной насадкой.

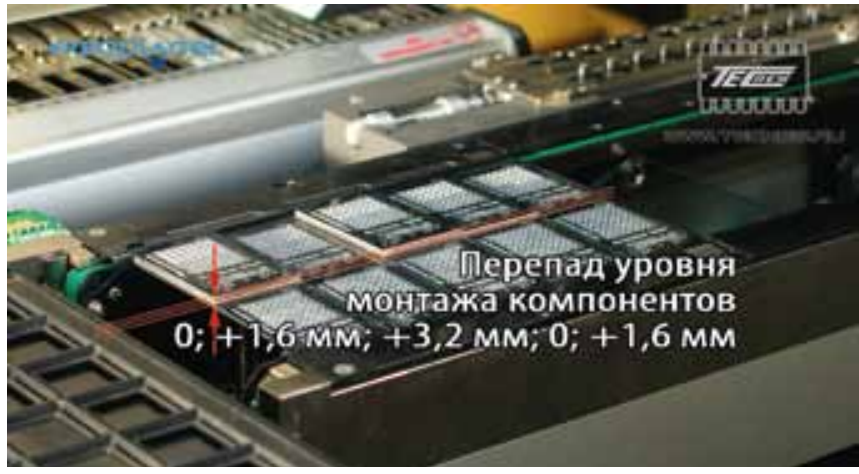

Рис. 5. Автоматическая компенсация перепада высоты установки

\section{ИЗМЕРЕНИЕ ЭЛЕКТРИЧЕСКИХ ПАРАМЕТРОВ КОМПОНЕНТОВ}

В условиях применения для сборки большого количества разнородных компонентов в процессе подготовки производства часто сказывается влияние человеческого фактора. При зарядке компонентов различных номиналов, но одного типоразмера, может быть совершена ошибка, которая в дальнейшем приведет к изготовлению целой партии неисправных изделий и, следовательно, к ее дорогостоящему ремонту или даже выбраковке. Также нельзя исключить возможность применения некачественных или контрафактных компонентов, когда электрические параметры не соответствуют указанным производителем допускам.

Дия предотвращения подобных ситуаций оборудование Europlacer оснащается специальным измерительным модулем. Модупь выполнен на базе поверяемого прибора

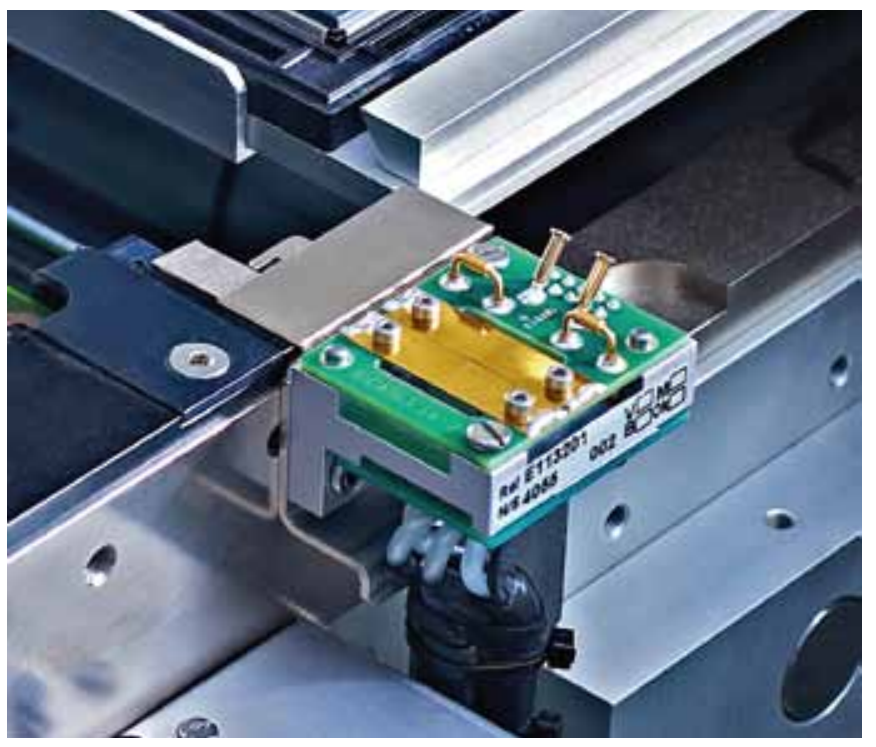

Рис. 6. Измерительный модуль на базе прибора НАMEC Instruments $\mathrm{GmbH}$ 
HAMEG Instruments GmbH. Автоматический контроль применяемых дия сборки изделия компонентов устраняет возможность ошибки оператора при загрузке компонентов. По выбору пользователя может проводиться контроль RLC-параметров всех чип-компонентов с сохранением результатов измерений дяя каждого изделия или частичный контроль при загрузке новых компоненmoв [4].

$$
\because * *
$$

Благодаря своим конструктивным особенностям установщики компонентов Europlacer могут обеспечить отечественным предприятиям ощутимые конкурентные преимущества. Проверенные решения уже применяются на многих предприятиях аэрокосмического и оборонного приборостроения, а также на предприятиях контрактной сборки электроники.

\section{ЛИТЕРАТУРА}

1. Чеснов М.Л., Курдюков Р. Ю., Зерний Ю. В. Основные проблемы автоматизации сборки в приборостроении // Вестник Московского государственного университета приборостроения и информатики. Серия. Приборостроение и информационные технологии. - М.: Изд-во МГУПИ, 2009. № 17. С. 56-61.

2. Балашов В.М., Добросельский М. А. Современные технологии производства при управлении качеством продукции: учебное пособие. - СПб: Изд-во ГУАП, 2007. 96 с.: ил.

3. ГОСТ IEC 61188-1-1-2013. Межгосударственный стандарт. Печатные платы и печатные узлы. Проектирование и применение. Часть 1-1. Общие требования. Приемлемая плоскостность для электронных сборок. - М.: Стандартинформ, 2014. 14 C.

4. Сайт Europlacer.ru [Электронный ресурс]. - Режим доступа: http://europlacer.ru/, свободный-(07.03.2020).

\section{НОВЫЕ КНИГИ ИЗДАТЕЛЬСТВА "ТЕХНОСФЕРА»}

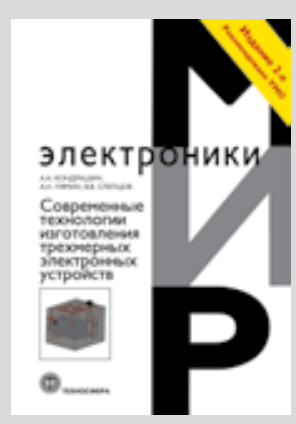

Цена 760 руб.

\section{СОВРЕМЕННЫЕ ТЕХНОЛОГИИ ИЗГОТОВЛЕНИЯ ТРЕХМЕРНЫХ ЭЛЕКТРОННЫХ УСТРОЙСТВ: Учеб. пособие. 2-е изд., испр. и доп.

\author{
Кондрашин А. А., Лямин А. Н., Спепцов В. В.
}

С развитием высоких технологий становится реальным выпуск трехмерных электронных устройств (ТЭУ). Решением данной задачи являются еще только разрабатываемые гибридные технологии, названные в данной работе квази-4D-технологиями формирования ТЭУ. В то же время создана классификация 4D-объектов (способных менять свою форму или структуру после их создания в зависимости от внешних условий, например при изменении температуры, при механическом воздействии и т.д.) ТЭУ и технологий для их формирования.

Данное учебное пособие является первой книгой по технологиям изготовления, сканирования и визуализации трехмерных электронных устройств. Во второй книге будут рассмотрены технологии сканирования трехмерных электронных устройств различных диапазонов, в том числе нанометрового диапазона. Отдельный раздел второй книги будет посвящен возможностям изготовления трехмерных электронных устройств нанометрового диапазона с применением методов сканирующей микроскопии. Третья книга будет посвящена технологиям визуализации (средствам отображения информации) для контроля параметров ТЭУ, создания новых ТЭУ и технологий реинжиниринга ТЭУ.

Учебное пособие может быть рекомендовано бакалаврам и магистрам высших учебных заведений. 
18-я МЕЖДУНАРОДНАЯ В ЫСТАВКА ЭЛЕКТРОНИКИ
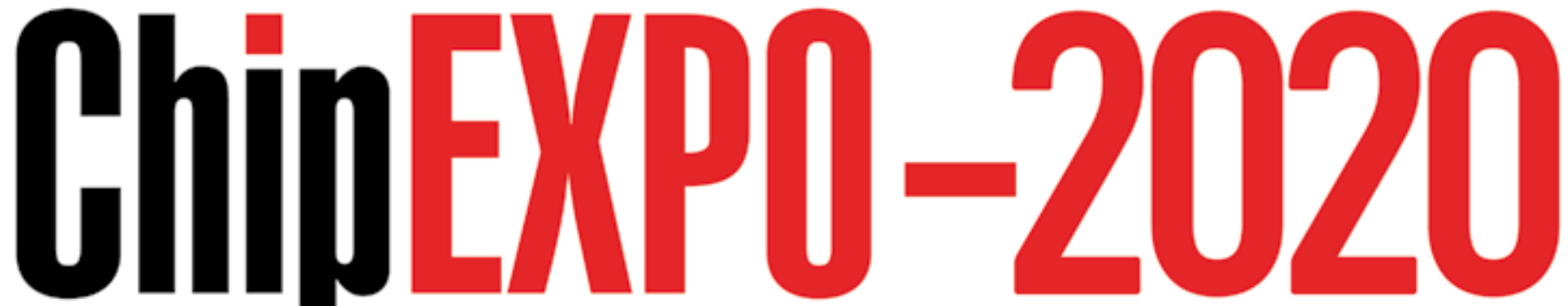

\section{КОМПОНЕНТЫ | ОБОРУДОВАНИЕ | ТЕХНОЛОГИИ}

\section{ВЫСТАВКА ПРОЙДЕТ}
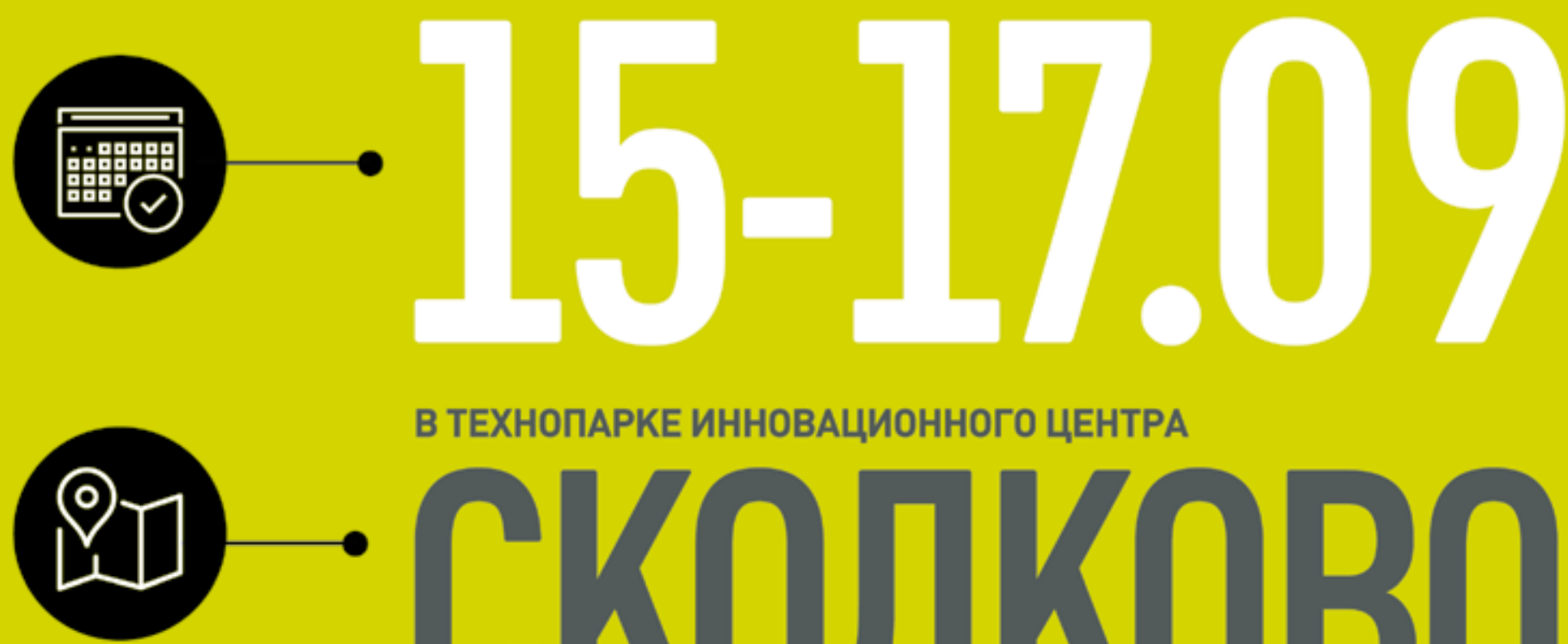

\section{В ТЕХНОПАРКЕ ИННОВАЦИОННОГО ЦЕНТРА}
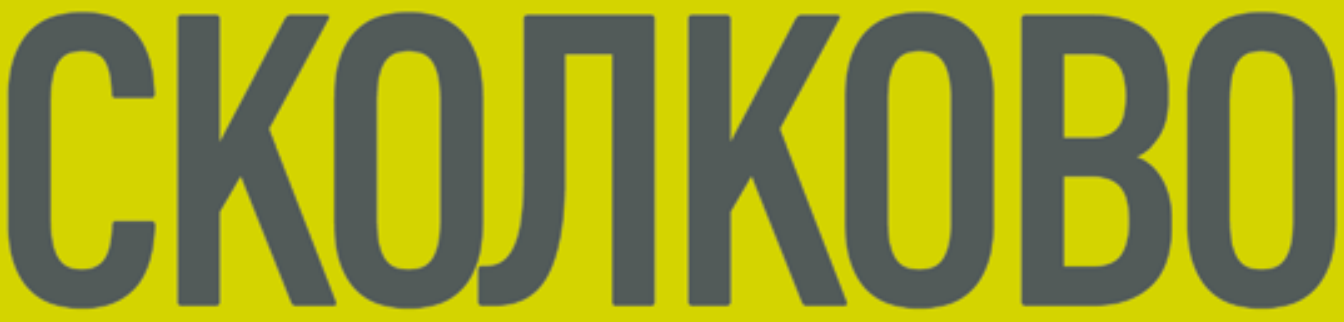

\section{ТЕМАТИЧЕСКИЕ ЭКСПОЗИЦИИ:}

- Экспозиция Департамента радиоэлектронной промышленности Минпромторга России, включая:

- экспозицию предприятий, являющихся изготовителями изделий, включенных в единый реестр российской радиоэлектронной продукции (Постановление Правительства РФ №878), - экспозицию разработок, созданных в рамках государственной программы

«Развитие электронной и радиоэлектронной промышленности на 2013-2025 годы»

[Постановление Правительства РФ №109],

- экспозицию разработок, обеспечивающих выполнение приоритетных национальных проектов.

- Дивизионы кластера

«Радиоэлектроника» ГК «Ростех» - Стартапы в электронике

- Квалифицированные поставщики ЭКБ

- Консорциумы и дизайн-центры по электронике

- Участники конкурса «Золотой Чип»

- Корпорация развития Зеленограда

ОФИЦИАЛЬНАЯ ПОДДЕРЖКА:
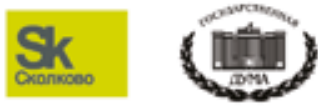

минпромторг 今 России

$\mathrm{O}_{\text {росэм }}$

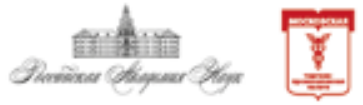

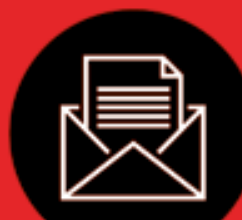

